\title{
Methylation status of the long control region of HPV 16 in clinical cervical specimens
}

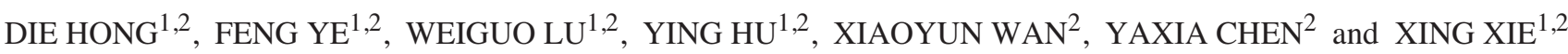 \\ ${ }^{1}$ Women's Reproductive Health Laboratory of Zhejiang Province, ${ }^{2}$ Department of Gynecologic Oncology, \\ Women's Hospital, School of Medicine, Zhejiang University, 2 Xueshi Road, Hangzhou 310006, P.R. China
}

Received January 7, 2008; Accepted March 5, 2008

\begin{abstract}
DNA methylation is one of the regulatory pathways that modulate human papillomavirus (HPV) gene expression. To obtain detailed methylation information on crucial areas of the long control region (LCR) of HPV 16 and to clarify the significance of methylation in clinical cervical lesions, 80 clinical samples were examined to determine the methylation status of the HPV 16 promoter and enhancer core using bisulfite modification and pyrosequencing. Seventy samples [26 of cervical carcinoma (CC), 13 of cervical intraepithelia neoplasia (CIN) III, 17 of CIN I-II and 14 of asymptomatic HPV 16 infection] were successfully examined. Analysis of the general methylation status of HPV 16 LCR in the 70 clinical specimens revealed $43(61.4 \%)$ with methylation in the promoter and/ or enhancer core of HPV 16. The proportion of methylated samples was highest in CC specimens (84.6\%), followed by asymptomatic infection (71.4\%) and CIN III (46.2\%), while the proportion of methylated samples was lowest in CIN I-II specimens (29.4\%). The methylation status of eight CpGs in HPV 16 LCR was determined in detail. In general, the methylation of $\mathrm{CpGs}$ was more common in the promoter than in the enhancer core region. The methylation frequencies of the eight CpGs ranged from $14.6 \pm 7.2$ to $33.7 \pm 23.0 \%$ in individual methylated $\mathrm{CpG}$ cases. The methylation pattern of all eight CpGs methylated in the promoter and enhancer core was more common in CC, and the pattern of scattered methylated CpGs was relatively more prevalent in asymptomatic infections. Our study demonstrates that DNA methylation is a common phenomenon in HPV 16 LCR clinical specimens, and may function as a host defense mechanism. While hypomethylation is probably associated with the initiation of neoplasia, hyper-
\end{abstract}

Correspondence to: Dr Xing Xie, Women's Reproductive Health Laboratory of Zhejiang Province and Department of Gynecologic Oncology, Women's Hospital, School of Medicine, Zhejiang University, 2 Xueshi Road, Hangzhou 310006, P.R. China

E-mail:xiex@mail.hz.zj.cn

Key words: human papillomavirus, methylation, pyrosequencing, cervical carcinoma, cervical intraepithelial neoplasia, long control region methylation in cervical cancer may be a reflection of the host defense mechanism. In the regulation of transcription, methylation is of more importance in the HPV 16 promoter than in the enhancer core.

\section{Introduction}

DNA methylation is a well recognized epigenetic mechanism in the regulation of gene expression (1-4). Methylation inhibits gene expression directly by interfering with transcription factor binding and/or indirectly by recruiting histone deacetylases through methyl-DNA-binding proteins (5). Studies have shown that DNA methylation is also an important regulatory pathway in the modulation of human papillomavirus (HPV) gene expression (6-9). In terms of transcription inhibition, the long control region (LCR) of HPV 16 is the region most influenced by DNA methylation.

It is generally known that the continued expression of oncogenic proteins E6 and E7 is necessary for malignant progression and for the maintenance of the transformed phenotype (10). The E6 and E7 genes of HPV 16 are transcribed from the promoter P97. P97 is under the control of an epithelial cell type-specific enhancer (11), whose activity is retained in the enhancer core (12). Thus, the promoter and enhancer core are the crucial regions of HPV 16 LCR. These regions contain several binding sites for cellular transcription factors such as TFIID (13), Sp1 (14), MSPF (8) and Tef-1 (15), which modulate its function positively, or YY1, which modulates it negatively (15), and the viral transcriptional regulatory protein E2 $(16,17)$. It has been confirmed in vivo that the methylation of the MSPF binding site suppresses the activity of the enhancer and of viral transcription (8). DNA methylation within the binding sites of transcription factors such as Sp1 might block binding indirectly, either by changing the conformation of chromatin or by interacting with methyl-CpG-specific repressor proteins (5). The methylation of E2 binding sites has been shown to prevent E2 from binding directly, whereas the HPV 16 E2 protein acts as a potent transcriptional activator of viral genes $(6,16)$.

The methylation status of HPV 16 LCR is associated with the transcription regulation of oncogenic genes in vivo. Using methods based on methylation-sensitive enzymes, direct Sanger sequencing or cloning and Sanger sequencing, researchers have detected the methylation status of HPV 16 LCR and other regions in clinical samples. In one study, 
Table I. PCR primer sequences.

\begin{tabular}{|c|c|c|}
\hline & Amplification primers $\left(5^{\prime}-3^{\prime}\right)$ & CpG position (nt) \\
\hline Enhancer primers & $\begin{array}{l}\text { Forward (bio): GTGTAATTATTGAATTATTATGT } \\
\text { Reverse: CACACACCCATATACAATTTTACA } \\
\text { Sequencing (reverse): ACCAAAAATATATACCTAAC }\end{array}$ & $7676,7682,7694$ \\
\hline Promoter primers & $\begin{array}{l}\text { Forward: TGTAAAATTGTATATGGGTGTGTG } \\
\text { Reverse (bio): ATCCTAAAACATTACAATTCTCTTTTAATA } \\
\text { Sequencing (forward): TTTATGTATAAAATTAAGGG }\end{array}$ & $31,37,43,52,58$ \\
\hline
\end{tabular}

which utilized enzymes and direct sequencing, more methylated samples were found in asymptomatic infections than in cervical cancer, indicating that hypomethylation is correlated with carcinogenic progression (18). However, another study using cloning and sequencing showed that methylation is more common in cervical cancer than in asymptomatic infections (19).

To some exent, the lack of consistency between these studies could be due to the use of assay and analysis procedures that could not quantify methylation adequately in the individual $\mathrm{CpG}$ sites of individual samples. However, a new technology, pyrosequencing, was recently established, which has the excellent advantage of quantification in individual sites. Initially, it was applied to the determination of allele frequencies of SNPs in pooled DNA samples (20). However, pyrosequencing has become a good method for analyzing methylation status with simultaneous quantification $(21,22)$, as the bisulfite treatment of DNA can convert epigenetic information into a genetic polymorphism.

There have been no reports with detailed information on HPV 16 LCR methylation by pyrosequencing in clinical samples, not even regarding the crucial regions of the promoter and enhancer core. The promoter P97 of HPV 16 contains potential methylation sites with five $\mathrm{CpG}$ dinucleotides located at $31,37,43,52$ and $58 \mathrm{nt}$. The enhancer core region contains three $\mathrm{CpG}$ dinucleotides located at 7677, 7683 and $7695 \mathrm{nt}$. These eight CpGs are the most influenced of all the HPV16 LCR CpGs $(9,19)$, and their methylation status represents the general methylation status of HPV 16 LCR.

In this study, we examined the methylation status of the HPV 16 promoter and enhancer core in clinical samples using bisulfite modification and pyrosequencing. Our aim was to obtain detailed methylation information on crucial regions of HPV 16 LCR, and to clarify the significance of methylation in clinical cervical lesions.

\section{Materials and methods}

Clinical samples. Cervical exfoliated cell samples were obtained from patients treated at the Women's Hospital, Medical School, Zhejiang University. We analyzed a panel of $80 \mathrm{HPV}$ 16-positive clinical specimens: 30 cases of cervical carcinoma (CC), 15 cases of cervical intraepithelia neoplasia (CIN) III, 18 cases of CIN I-II and 17 cases of asymptomatic HPV 16 infection. $\mathrm{CC}$ and its precursors were confirmed by histology, while asymptomatic infections were confirmed by the thinlayer cytology test. HPV genotype detection was performed with PCR-RFLP as previously described (23). Informed consent for the study was obtained.

Bisulfite modification. The cytosine methylation status of the HPV16 DNA was determined by bisulfite modification of the target DNA. Sodium bisulfite conversion of genomic DNA was performed as previously described (24), including alkaline denaturation, deamination, desulfonation, neutralization and desalting. Sample DNA (50-2,000 ng) supplemented with $1 \mu \mathrm{g}$ of salmon sperm DNA in a total volume of $18 \mu 1$ in water was denatured with $2 \mu 1$ of $3 \mathrm{M} \mathrm{NaOH}$ and incubated at $37^{\circ} \mathrm{C}$ for $20 \mathrm{~min}$. Thereafter, $150 \mu \mathrm{l}$ freshly prepared bisulfite was added directly to the denatured DNA. The mixture was incubated in a thermal cycler at $99^{\circ} \mathrm{C}$ for $5 \mathrm{~min}, 60^{\circ} \mathrm{C}$ for $25 \mathrm{~min}$, $99^{\circ} \mathrm{C}$ for $5 \mathrm{~min}, 60^{\circ} \mathrm{C}$ for $85 \mathrm{~min}, 99^{\circ} \mathrm{C}$ for $5 \mathrm{~min}$ and $60^{\circ} \mathrm{C}$ for 175 min. The modified DNA was desalted with the Wizard DNA Clean-Up System and subsequently desulfonated by the addition of $\mathrm{NaOH}$ for a final concentration of $0.3 \mathrm{M}$, then incubated at $37^{\circ} \mathrm{C}$ for $20 \mathrm{~min}$. The DNA was precipitated with $47 \mu 1$ of $10 \mathrm{M}$ ammonium acetate and $500 \mu \mathrm{l}$ of $100 \%$ ethanol at $-20^{\circ} \mathrm{C}$ overnight. The pellet was washed with $70 \%$ ethanol and dissolved in $20 \mu \mathrm{l}$ TE buffer (1 mM Tris-HCl, $\mathrm{pH} 8.0$ ).

PCR and pyrosequencing. The promoter region and enhancer core region were examined separately for $\mathrm{CpG}$ methylation status under the same conditions.

PCR was performed in a $50-\mu 1$ reaction mixture containing 10X PCR buffer, $25 \mathrm{mM} \mathrm{MgCl} 2,200 \mu \mathrm{M}$ dNTP, 2 U Taq polymerase, $10 \mathrm{pmol}$ of each primer (Table I) and bisulfitemodified DNA. PCR conditions were as follows: preheating at $94^{\circ} \mathrm{C}$ for $5 \mathrm{~min}, 40$ cycles at $94^{\circ} \mathrm{C}$ for $45 \mathrm{sec}, 55^{\circ} \mathrm{C}$ for $45 \mathrm{sec}$, $72^{\circ} \mathrm{C}$ for $45 \mathrm{sec}$, and a final extension at $72^{\circ} \mathrm{C}$ for $10 \mathrm{~min}$. One of the primers in each amplification reaction was labeled with 5-biotin. The choice of primer for 5-biotinylation was based on the direction of pyrosequencing (reverse primer biotinylated for forward sequencing and forward primer biotinylated for reverse sequencing) (25).

PCR amplifiers were detected by pyrosequencing to identify the methylation frequency of cytosine residues within HPV 16 DNA. Purification with streptavidin Sepharose HP beads and the codenaturation of the biotinylated PCR products and sequencing primer were conducted following the PSQ 96 sample preparation introduction, then subjected to sequencing using an automatically generated nucleotide dispensation order for 'sequences to analyze'. The pyrograms were analyzed using the allele quantification mode to determine the proportion of $\mathrm{C} / \mathrm{T}$ (or $\mathrm{G} / \mathrm{A}$ ) at the targeted position(s). If the sequencing 

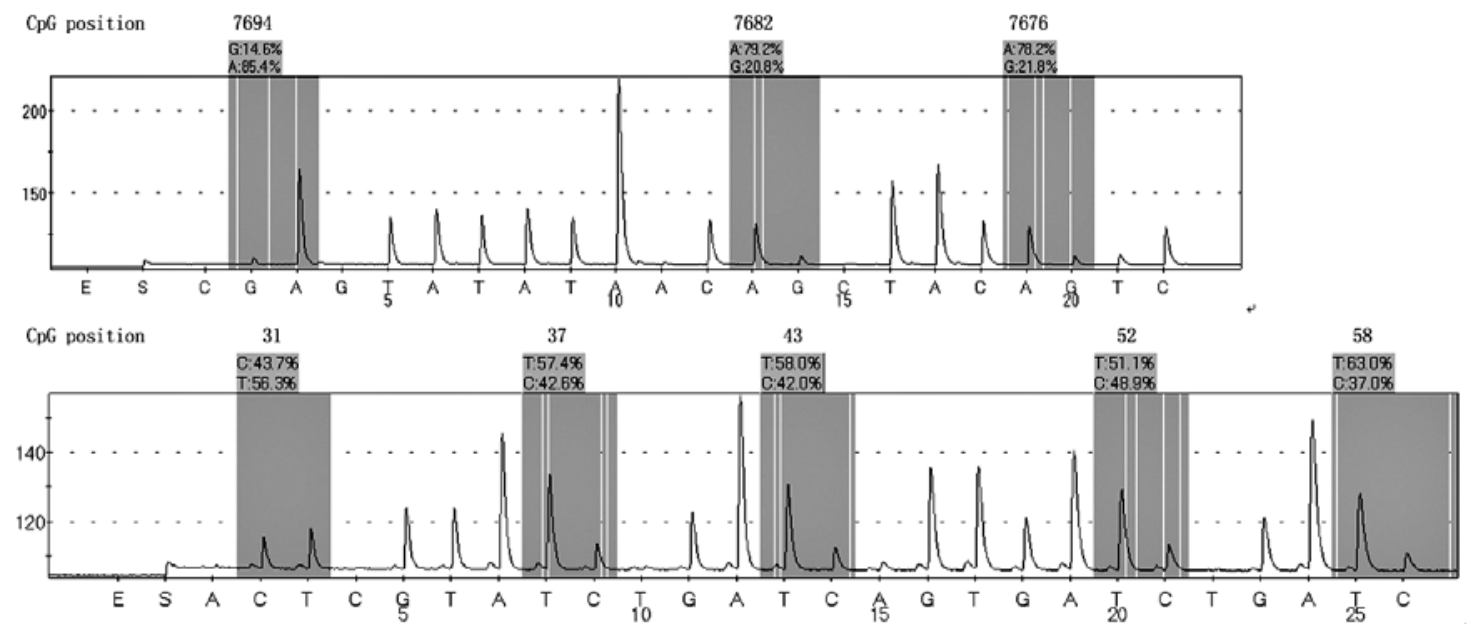

Figure 1. Representative pyrograms quantifying methylation levels at CpG sites in the HPV 16 promoter and enhancer core region. CpG nucleotide positions are 7676, 7682 and 7694 nt in the enhancer core, and 31,37, 43, 52 and 58 nt in the promoter. The sequencing primer of the enhancer region was reversed; the sequence acquired an antisense strand. The frequency of $\mathrm{G}$ in this antisense strand is equal to $\mathrm{C}$ in the sense strand.

Table II. Proportion of samples with methylated HPV16 promoter or enhancer core in clinical cervical specimens $(n=70)$.

\begin{tabular}{lccc}
\hline & \multicolumn{2}{c}{ Methylation status $^{\mathrm{a}}$} & \\
\cline { 2 - 3 } Group & $\begin{array}{c}\text { Methylated } \\
\text { no. }(\%)\end{array}$ & $\begin{array}{c}\text { Unmethylated } \\
\text { no. }(\%)\end{array}$ & $\begin{array}{c}\text { Total } \\
\text { no. }\end{array}$ \\
\hline $\begin{array}{l}\text { Asymptomatic } \\
\text { infection }\end{array}$ & $10(71.4)$ & $4(28.6)$ & 14 \\
CIN I-II & $5(29.4)$ & $12(70.6)$ & 17 \\
CIN III & $6(46.2)$ & $7(53.8)$ & 13 \\
CC & $22(84.6)$ & $4(15.4)$ & 26 \\
Total & $43(61.4)$ & $27(38.6)$ & 70 \\
\hline
\end{tabular}

${ }^{a}$ Methylation status: $\chi^{2}=15.125, \mathrm{P}=0.002$ ( $\chi^{2}$ test). CIN, cervical intraepithelial neoplasia; $\mathrm{CC}$, cervical carcinoma.

primer was forward, the targeted site was $\mathrm{C} / \mathrm{T}$, whereas if the sequencing primer was reversed, the targeted site was G/A. The frequency of $\mathrm{G}$ in the antisense strand was equal to $\mathrm{C}$ in the sense strand, representing the methylation frequency in the targeted $\mathrm{CpG}$ site of an individual sample.

Statistical analysis. For statistical analysis, the $\chi^{2}$ test and the Kruskal-Wallis test were used. P-values $<0.05$ were considered statistically significant, and all tests were two-sided.

\section{Results}

General methylation status of HPV 16 LCR in clinical cervical specimens. Bisulfite modification and pyrosequencing were used to detect the CpG methylation status of HPV 16 LCR in clinical specimens (Fig. 1). The complete conversion of $\mathrm{C}$ to $\mathrm{T}$ in a non-CpG site was identified in order to ensure the successful modification of bisulfite. CpGs in the promoter (positions $31,37,43,52$ and $58 \mathrm{nt}$ ) and in the enhancer core (nucleotide positions 7676, 7682 and $7694 \mathrm{nt}$ ) were detected to exhibit the methylation status of HPV 16 LCR. C and T (or G and A in
Table III. Particular methylation status of eight CpGs in the HPV 16 LCR of clinical cervical specimens $(n=70)$.

\begin{tabular}{ccc}
\hline CpG site (nt) & $\begin{array}{c}\text { No. of methylated } \\
\text { cases }^{\mathrm{a}}\end{array}$ & $\begin{array}{c}\text { Methylation } \\
\text { frequency }\end{array}$ \\
\hline 7676 & 14 & $23.8 \pm 15.7$ \\
7682 & 15 & $15.7 \pm 10.3$ \\
7694 & 8 & $14.6 \pm 7.2$ \\
31 & 41 & $29.2 \pm 22.8$ \\
37 & 38 & $32.9 \pm 23.3$ \\
43 & 38 & $33.7 \pm 23.0$ \\
52 & 39 & $33.0 \pm 24.4$ \\
58 & 36 & $32.0 \pm 21.9$
\end{tabular}

${ }^{\mathrm{a}} \chi^{2}=77.773, \mathrm{P}=0.000$ ( $\chi^{2}$ test). ${ }^{\mathrm{b}}$ Methylation frequency of the methylated cases. $\chi^{2}=16.266, \mathrm{P}=0.023$ (Kruskal-Wallis test).

the antisense strand) indicated the corresponding frequencies of methylated and unmethylated cytosines at each $\mathrm{CpG}$ site (Fig. 1, shaded grey region).

Out of 80 samples, examination of the promoter or enhancer regions was unsuccessful in 10 . These were therefore excluded from the statistical analysis, and 70 samples were analyzed for the methylation status of HPV 16 LCR.

Forty-three samples showed some degree of methylation in CpGs in the promoter and/or enhancer core regions of HPV 16. The residual 27 samples were unmethylated. The proportion of methylated samples was highest in CC $(84.6 \%)$, followed by asymptomatic infection (71.4\%) and CIN III (46.2\%). It was lowest in CIN I-II (29.4\%) (Table II).

Methylation status of eight CpGs in the HPV 16 LCR of clinical cervical specimens. The methylation status of each $\mathrm{CpG}$ in HPV 16 LCR differed among the clinical cervical specimens (Table III). Generally, the methylation of CpGs was more common in the promoter than in the enhancer core region. On the one hand, more samples showed methylation in CpGs in the HPV 16 promoter. Of all 70 clinical cervical specimens, 41, 38, 
Table IV. Proportion of samples with different methylated CpG distribution within the HPV16 promoter and enhancer core in clinical cervical specimens $(\mathrm{n}=70)$.

\begin{tabular}{|c|c|c|c|c|c|c|c|}
\hline Group & $\begin{array}{c}\text { Total } \\
\text { no. }(\%)\end{array}$ & $\begin{array}{l}\text { Asymptomatic } \\
\text { infection no. }(\%)\end{array}$ & $\begin{array}{l}\text { CIN I-II } \\
\text { no. }(\%)\end{array}$ & $\begin{array}{l}\text { CIN III } \\
\text { no. }(\%)\end{array}$ & $\begin{array}{c}\mathrm{CC} \\
\text { no. }(\%)\end{array}$ & $\chi^{2}$ & P-value \\
\hline All CpGs methylated & $8(11.4)$ & 0 & 0 & $1(1.4)$ & $7(10.0)$ & 10.346 & 0.016 \\
\hline Methylated promoter $\mathrm{CpGs}^{\mathrm{a}}$ & $27(38.6)$ & $5(7.1)$ & $5(7.1)$ & $5(7.1)$ & $12(17.1)$ & 1.281 & 0.734 \\
\hline Methylated enhancer $\mathrm{CpGs}^{\mathrm{b}}$ & 0 & 0 & 0 & 0 & 0 & - & - \\
\hline Scattered methylated CpGs & $8(11.4)$ & $5(7.1)$ & 0 & 0 & $3(4.3)$ & 12.029 & 0.007 \\
\hline No methylated CpGs & $27(38.6)$ & $4(5.7)$ & $12(17.1)$ & $7(10.0)$ & $4(5.7)$ & 15.125 & 0.002 \\
\hline Total & 70 & 14 & 17 & 13 & 26 & & \\
\hline
\end{tabular}

${ }^{a}$ Methylated promoter CpGs include samples with all five CpGs methylated in the promoter, but exclude samples with all CpGs methylated. ${ }^{b}$ Methylated enhancer CpGs include samples with three CpGs methylated in the enhancer core, but exclude samples with all CpGs methylated. CIN, cervical intraepithelial neoplasia; $\mathrm{CC}$, cervical carcinoma.

38,39 and 36 cases were methylated in the promoter region at CpG-31 nt, CpG-37 nt, CpG-43 nt, CpG-52 nt and CpG-58 nt, respectively. Only 14,15 and 8 cases were methylated in the enhancer core region at CpG-7676 nt, CpG-7682 nt and CpG$7694 \mathrm{nt}$, respectively. There was a statistical difference between the eight $\mathrm{CpGs}$ regarding the number of methylated cases. However, there were no differences between the five CpGs in the promoters. On the other hand, among these methylated cases of individual $\mathrm{CpGs}$, the methylation frequency was also higher at $\mathrm{CpGs}$ in the promoter than in the enhancer core. Concerning methylation frequency, a statistical difference among the eight CpGs was also exhibited. No CpGs had a methylation frequency of $100 \%$ in all the clinical specimens.

Methylation patterns of HPV 16 LCR in clinical cervical specimens. Methylation patterns differed among the clinical cervical specimens. Some samples had a methylation pattern of all eight CpGs methylated in the promoter and enhancer core. Some exhibited a pattern of all five CpGs methylated in the promoter, in spite of the methylation status of the enhancer (excluding all eight methylated $\mathrm{CpGs}$ ), while other specimens had only scattered methylated CpGs in the promoter and/or enhancer regions. No samples had a pattern of all three CpGs methylated in the enhancer core, despite the methylation status of the enhancer (excluding all eight methylated CpGs). The methylation patterns differed significantly among the cervical lesion groups (Table IV). Of the 70 samples, 8 (11.4\%) displayed a methylation pattern of all $\mathrm{CpGs}$ methylated, and 7 $(10 \%)$ of these 8 were found in CC. Eight samples $(11.4 \%)$ showed a methylation pattern of scattered methylated $\mathrm{CpG}$, with $5(7.1 \%)$ of these 8 in asymptomatic HPV infection.

\section{Discussion}

The transcription of HPV 16 oncogenes is influenced by several factors. DNA methylation provides an additional means of regulating HPV transcription. Several studies have tried to investigate methylation status in clinical cervical lesions. Badal et al (18) showed that $24.7 \%$ (20/81) of cases were methylated in the LCR and E6 gene of HPV 16 in clinical samples, while Kalantari et al (19) detected 64.3\% (74/115) in HPV 16 LCR and Turan et al (26) 48.6\% (18/37) in HPV 18 LCR. In accordance with some of these studies, our data showed that HPV 16 LCR could be targeted efficiently by epithelial $\mathrm{CpG}$ methylation machinery. By examining eight CpGs in the promoter and enhancer core of HPV 16 LCR using bisulfite modification and pyrosequencing, we found that $61.4 \%$ (43/70) of the clinical cervical specimens had methylated CpGs in HPV 16 LCR. This phenomenon probably occurs as a result of the host cellular defense mechanism $(3,27)$. In HPV infection specimens, the expression of oncogenic proteins E6 and E7 often has the potential to induce the malignant transformation of host cells (10). Some negative regulatory pathways, such as DNA de novo methylation, may be activated by host cells as a defense against the activation of oncogenic proteins (3).

It is well known that the regions of the HPV 16 promoter and enhancer core can be combined by many transcription factors $(8,14,15)$. DNA methylation and the accompanying compact chromatin configuration of the regions can block the binding of the transcription factors directly or indirectly, ultimately resulting in transcription repression $(5,6,8)$. In our study, we found that methylation of CpGs was more common in the HPV 16 promoter than in the enhancer core, and that the methylation frequency of these methylated CpGs was also higher in the promoter. The data indicate that the methylation of the HPV 16 promoter is more crucial than that of the enhancer. The failure of a combination of Sp1 or other transcription factors in the promoter due to DNA methylation is probably more vital. On the other hand, no differences were found between the five $\mathrm{CpG}$ in the promoter, which indicated a collective effect of DNA methylation in a small region.

The normal HPV 16 life cycle is mostly restricted to asymptomatic infections and CIN (especially CIN I-II), while carcinogenic progression is a chance occurrence (18). Our study showed that, in the stage of asymptomatic infection, DNA methylation of HPV 16 LCR was a common phenomenon, while the proportion of methylated samples decreased markly in CIN I-II. Of 14 cases of asymptomatic infection, 10 showed methylation in the CpGs of the promoter and/or enhancer core. However, only 5 out of 17 cases of CIN I-II showed methylation. Another study (19), using sequencing, showed 
that 36 of 51 cases were methylated in asymptomatic infection samples and 7 of 17 cases were methylated in CIN. It was suggested that HPV 16 commonly exists as an episomal form in asymptomatic infections and CIN I-II. The high prevalence of methylated HPV 16 LCR in asymptomatic epithelia may indicate that methylation is part of normal HPV 16 biology. DNA methylation may be one of the numerous strategies developed by HPVs that favors a subclinical long-term maintenance of the viral infection (18). The low methylation levels of HPV genomes in CIN I-II lesions, where HPV 16 normally replicates episomally, may stem from the expansion of the transcriptionally active cell population, as expected from the initiation of the neoplastic process and an increase in the virus genomes (28). However, in regard to the unmethylated samples in the asymptomatic infection, it is unknown whether they are so before clearance, or whether they are convenient for the initiation of neoplasia.

In agreement with studies on cervical lesions and oral carcinoma $(19,9)$, our study showed that the methylation of HPV 16 LCR was most common in cervical cancer. The prevalence of methylated HPV 16 LCR in cervical cancer seems paradoxical in relation to the DNA methylation mechanism, which is generally known to lead to transcriptional repression. In fact, the expression of E6 and E7 in each cervical cancer cell could be very high. Expression of these oncoproteins not only relates to methylation status, but also to the copy number of the virus per cell (25). On the one hand, a higher frequency of HPV 16 LCR ought to inhibit more downstream gene expression of an individual cell, but the methylation frequency of HPV 16 LCR is no more than $50 \%$ in cervical cancer specimens, insufficient to repress transcription completely. On the other hand, more HPVs are inserted into the host genome with the progression of cervical lesions (29). Generally, hundreds of HPV copies are inserted into the genome of an individual cervical cancer cell. In an individual cell with a majority of copies of HPV 16, even if the transcription efficiency of each HPV copy is low, the total oncoproteins E6 and E7 in a cell could be high overall. For example, the methylation frequency of HPV 16 in CaSki cells is very high, but the cells contain 500 copies of HPV 16. Expression of E6 and E7 is still 2.5-fold in CaSki cells compared to SiHa cells (30), which contain a copy of nearly unmethylated HPV 16 (25).

Though the frequency of DNA methylation likely has no negative linear relation to the expression of E6 and E7 in CC and CIN III, the methylation reflects the insertion of viral genes and the defense mechanism of the host cells. It is known that viral infections, particularly upon the insertion of viral genes into host genomes, can trigger host defense mechanisms such as methylation machinery activation (31). Methylation of HPV LCR probably comes from tandem repetition and chromosomal integration of HPV 16 DNA (19). To some degree, the methylation pattern of HPV 16 LCR is also a reflection of the host defense mechanism, and the presence of HPV may be associated with the methylation pattern. It is probable that, in the course of integration, CpGs in HPV 16 LCR can be easily and simultaneously targeted by the host defense mechanism while, in episomal form, it is more common for $\mathrm{CpG}$ to have eterogeneous methylated status in HPV 16 LCR.

Kim et al reported that HPV 16 LCR was selectively hypomethylated in highly differentiated cell populations and relatively hypermethylated in poorly differentiated cells (6). Our study also tried to analyze the association between HPV LCR methylation status and cell differentiation status among the 26 cases of CC (data not shown). However, most of the cases recruited were well- or moderately-differentiated, making statistical analysis difficult. However, our study still demonstrated that DNA methylation of HPV 16 LCR was a common phenomenon in a well- or moderately-differentiated cell population of clinical cervical cancer specimens.

In conclusion, our study demonstrated a common phenomenon of DNA methylation in HPV 16 LCR in clinical specimens, a phenomenon which may function as a host defense mechanism. Our data show that the methylation of HPV 16 LCR was prevalent in CC and asymptomatic infection, but uncommon in CIN, especially in CIN I-II. Hypomethylation is probably associated with the initiation of neoplasia, while hypermethylation in CC may be the reflection of the host's defenses. In the regulation of transcription, methylation is more crucial in the HPV 16 promoter than in the enhancer core

\section{Acknowledgements}

We are grateful to members of the Department of Gynecologic Oncology, Women's Hospital, School of Medicine, Zhejiang University, for access to and assistance with the sample collection. Support for our research is provided by the Research Fund for the Doctoral Program of Higher Education (20070335054) and the Natural Science Fund of Zhejiang Province (Y205190).

\section{References}

1. Fuks F: DNA methylation and histone modifications: teaming up to silence genes. Curr Opin Genet Dev 15: 490-495, 2005.

2. Bird A: DNA methylation patterns and epigenetic memory. Genes Dev 16: 6-21, 2002.

3. Doerfler W: On the biological significance of DNA methylation. Biochemistry 70: 505-524, 2005.

4. Mino A, Onoda N, Yashiro M, et al: Frequent p16 CpG island hypermethylation in primary remnant gastric cancer suggesting an independent carcinogenic pathway. Oncol Rep 15: 615-620, 2006.

5. Zhu WG, Srinivasan K, Dai Z, et al: Methylation of adjacent $\mathrm{CpG}$ sites affects $\mathrm{Sp} 1 / \mathrm{Sp} 3$ binding and activity in the p21(Cip1) promoter. Mol Cell Biol 23: 4056-4065, 2003.

6. Kim K, Garner-Hamrick PA, Fisher C, Lee D and Lambert PF: Methylation patterns of papillomavirus DNA, its influence on E2 function, and implications in viral infection. J Virol 77: 12450-12459, 2003.

7. Thain A, Jenkins O, Clarke AR and Gaston K: CpG methylation directly inhibits binding of the human papillomavirus type $16 \mathrm{E} 2$ protein to specific DNA sequences. J Virol 70: 7233-7235, 1996.

8. List HJ, Patzel V, Zeidler U, et al: Methylation sensitivity of the enhancer from the human papillomavirus type 16 . J Biol Chem 269: 11902-11911, 1994.

9. Balderas-Loaeza A, Anaya-Saavedra G, Ramirez-Amador VA, et al: Human papillomavirus-16 DNA methylation patterns support a causal association of the virus with oral squamous cell carcinomas. Int J Cancer 120: 2165-2169, 2007.

10. Munger K, Baldwin A, Edwards KM, et al: Mechanisms of human papillomavirus-induced oncogenesis. J Virol 78: 11451-11460, 2004

11. Cripe TP, Haugen TH, Turk JP, et al: Transcriptional regulation of the human papillomavirus-16 E6-E7 promoter by a keratinocyte-dependent enhancer, and by viral E2 transactivator and repressor gene products: implications for cervical carcinogenesis. EMBO J 6: 3745-3753, 1987.

12. Cripe TP, Alderborn A, Anderson RD, et al: Transcriptional activation of the human papillomavirus-16 P97 promoter by an 88-nucleotide enhancer containing distinct cell-dependent and AP-1-responsive modules. New Biol 2: 450-463, 1990. 
13. Dostatni N, Lambert PF, Sousa R, Ham J, Howley PM and Yaniv M: The functional BPV-1 E2 transactivating protein can act as a repressor by preventing formation of the initiation complex. Genes Dev 5: 1657-1671, 1991 .

14. Tan SH, Gloss B and Bernard HU: During negative regulation of the human papillomavirus-16 E6 promoter, the viral E2 protein can displace Sp1 from a proximal promoter element. Nucleic Acids Res 20: 251-256, 1992.

15. Turek LP: The structure, function, and regulation of papillomaviral genes in infection and cervical cancer. Adv Virus Res 44: 305-356, 1994.

16. Bouvard V, Storey A, Pim D and Banks L: Characterization of the human papillomavirus E2 protein: evidence of transactivation and transrepression in cervical keratinocytes. EMBO J 13: $5451-5459,1994$

17. Lambert PF, Dostatni N, McBride AA, Yaniv M, Howley PM and Arcangioli B: Functional analysis of the papilloma virus E2 transactivator in Saccharomyces cerevisiae. Genes Dev 3: 38-48, 1989.

18. Badal V, Chuang LS, Tan EH, et al: CpG methylation of human papillomavirus type 16 DNA in cervical cancer cell lines and in clinical specimens: genomic hypomethylation correlates with carcinogenic progression. J Virol 77: 6227-6234, 2003.

19. Kalantari M, Calleja-Macias IE, Tewari D, et al: Conserved methylation patterns of human papillomavirus type 16 DNA in asymptomatic infection and cervical neoplasia. J Virol 78: 12762-12772, 2004.

20. Alderborn A, Kristofferson A and Hammerling U: Determination of single-nucleotide polymorphisms by real-time pyrophosphate DNA sequencing. Genome Res 10: 1249-1258, 2000.
21. Wong HL, Byun HM, Kwan JM, et al: Rapid and quantitative method of allele-specific DNA methylation analysis. Biotechniques 41: 734-739, 2006.

22. Dupont JM, Tost J, Jammes H and Gut IG: De novo quantitative bisulfite sequencing using the pyrosequencing technology. Anal Biochem 333: 119-127, 2004.

23. Hong D, Ye F, Chen H, et al: Distribution of human papillomavirus genotypes in the patients with cervical carcinoma and its precursors in Zhejiang Province, China. Int J Gynecol Cancer 18: 104-109, 2008.

24. Grunau C, Clark SJ and Rosenthal A: Bisulfite genomic sequencing: systematic investigation of critical experimental parameters. Nucleic Acids Res 29: E65-E65, 2001

25. Rajeevan MS, Swan DC, Duncan K, Lee DR, Limor JR and Unger ER: Quantitation of site-specific HPV 16 DNA methylation by pyrosequencing. J Virol Methods 138: 170-176, 2006.

26. Turan T, Kalantari M, Calleja-Macias IE, et al: Methylation of the human papillomavirus-18 L1 gene: a biomarker of neoplastic progression? Virology 349: 175-183, 2006.

27. Galagan JE and Selker EU: RIP: the evolutionary cost of genome defense. Trends Genet 20: 417-423, 2004.

28. Stoler MH, Rhodes CR, Whitbeck A, Wolinsky SM, Chow LT and Broker TR: Human papillomavirus type 16 and 18 gene expression in cervical neoplasias. Human Pathol 23: 117-128, 1992.

29. Furumoto $\mathrm{H}$ and Irahara M: Human papilloma virus (HPV) and cervical cancer. J Med Invest 49: 124-133, 2002.

30. Rajeevan MS, Swan DC, Nisenbaum R, et al: Epidemiologic and viral factors associated with cervical neoplasia in HPV-16positive women. Int J Cancer 115: 114-120, 2005.

31. Verma M: Viral genes and methylation. Ann NY Acad Sci 983: 170-180, 2003. 